\title{
Gravity as an Environmental System
}

Theodore W. Hall

Chinese University of Hong Kong 



\title{
Gravity as an Environmental System
}

\author{
Theodore W. Hall \\ Chinese University of Hong Kong
}

Copyright (@ 2000 Society of Automotive Engineers, Inc.

\begin{abstract}
This paper examines gravity as a controllable environmental parameter. In long-duration orbital habitats and interplanetary vessels, designers face difficult decisions as to whether to provide artificial gravity, and if so, how much. Habitats on the moon and minor planets pose other issues. Partial gravity offers greater freedom of movement on the vertical axis, but may hinder floor traction and horizontal mobility. The minimum gravity required to preserve health remains unknown. Supplementing significant but insufficient natural gravity with artificial gravity is problematic. However, the presence of at least some natural gravity may offer other alternatives that are not available in orbit.
\end{abstract}

\section{INTRODUCTION}

In the nearly 40 years of manned space flight, the state of gravity has been taken as a given. Like the weather, it has been beyond control. Moreover, except for a few days on the lunar surface, shared by only twelve people, humanity's extraterrestrial experience has been entirely in microgravity. Consequently, there has been an all-or-nothing approach with respect to gravitational design. Engineers and architects have invested much effort in adapting orbital habitats for microgravity, while conceiving planetary habitats essentially the same as if they were destined for a desert on Earth.

As humans move beyond Earth in greater numbers for longer periods of time, it behooves designers to take a more proactive role, not only in reacting to the particular state of gravity in a particular place, but also in choosing it. This paper explores gravity as a controllable environmental parameter, on a par with light, heat, humidity, ventilation, and other aspects of indoor climate.

It has been said that everybody complains about the weather, but nobody ever does anything about it. This is, of course, not true. People routinely construct enclosed environments with controlled climates. When nature provides too much of something, they screen it out. When nature provides too little, they supplement it through engineered environmental systems. In most cases, the "natural" (ambient) and "artificial" (engineered) values are not in opposition, but are rather orthogonal, independent values. Designers typically engineer the artificial value to supplement what nature provides.

For example: natural daylight may be adequate for working near a window, but interior spaces may require additional artificial lighting from incandescent or fluorescent lamps. As daylight decreases, one may switch on more lamps, or increase their intensity by adjusting rheostats. One need not wait for the complete absence of natural light before switching on some lamps. Similarly, people typically employ electric or combustion heaters well before the outside temperature reaches absolute zero.

The question is whether gravity can be similarly supplemented, controlled, and adjusted.

\section{PHENOTYPES OF GRAVITY ENVIRONMENTS}

To date, the gravitational aspects of virtually all environmental designs fall into three major phenotypes: natural planetary gravity, micro gravity, and artificial gravity. Natural and artificial gravity may appear to be mutually exclusive, at opposite ends of a continuum, each fading through an indistinct boundary into a micro-gravity midpoint. Figure 1 illustrates this concept.

This is not a particularly useful image if one wishes to speak of gravitational systems, with gravity as a controllable parameter.

\begin{tabular}{|c|c|c|c|c|}
\hline 1.00 & 0.38 & 0.170 & 0.38 & 1.00 \\
\hline Earth & Mars & $\begin{array}{l}\text { Moon } \\
\text { Salyut } \\
\text { Skylab } \\
\text { Mir } \\
\text { ISS }\end{array}$ & Mars transit & $\begin{array}{l}\text { Stanford Torus } \\
\text { Bernal Sphere }\end{array}$ \\
\hline & & Micro & Artifi & ial \\
\hline
\end{tabular}

Figure 1: Natural and Artificial Gravity as Mutually Exclusive 


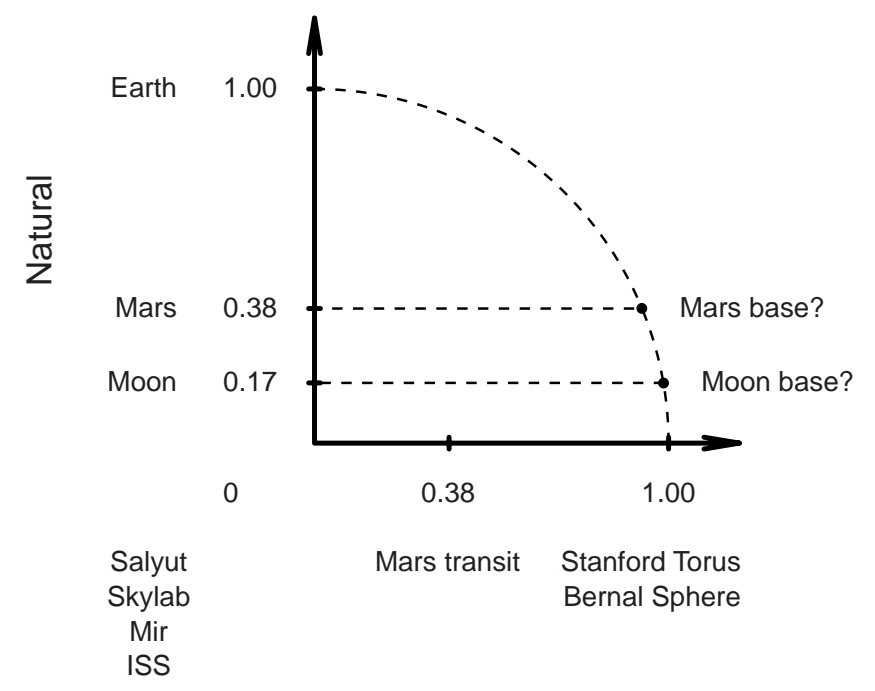

Artificial

\section{Figure 2: Natural and Artificial Gravity as Orthogonal} Components

Figure 2 represents natural and artificial gravity as orthogonal values. Viewed in this way, extraterrestrial designs have clung to the axes; the open space between the axes is largely unexplored.

To examine the merits of this view, it's necessary first to characterize the natural and artificial components, as well as the null vector (micro gravity), and consider whether there's any advantage in trying to supplement one component with the other.

In the equations that follow, boldface symbols represent vectors, while italic symbols represent scalar magnitudes. Units of measure are kilograms $(\mathrm{Kg})$, meters $(\mathrm{m})$, seconds $(\mathrm{s})$, and radians (dimensionless).

NATURAL GRAVITY - Mutual gravitational attraction between particles of mass is an innate quality of matter. There is no known way to either magnify or insulate against this attraction, other than by varying the masses of the particles or the distance between them.

People's common experience of weight arises not from the attraction as such, but rather from resistance to it, offered by the solid ground, the elevator cable, the airplane wing, or whatever structure holds them up. Should that structure fail, allowing them to follow the attraction unimpeded, the benefits of gravity would quickly diminish.

The natural gravitational acceleration at the surface of a planet is proportional to the planet's average density and radius:

$$
\begin{aligned}
A_{\mathrm{g}} & =\mathrm{G} \cdot \mathrm{m} / \mathrm{r}^{2} \\
& =\mathrm{G} \cdot \rho \cdot(4 / 3) \cdot \pi \cdot r^{3} / r^{2} \\
& =4 \cdot \pi \cdot \mathrm{G} \cdot \rho \cdot r / 3
\end{aligned}
$$

where $A_{\mathrm{g}}$ is the gravitational acceleration $\left(\mathrm{m} / \mathrm{s}^{2}\right), \mathrm{G}$ is the gravitational constant $\left(6.673 \cdot 10^{-11} \cdot \mathrm{m}^{3} /\left(\mathrm{Kg} \cdot \mathrm{s}^{2}\right)\right), m$ is the planet's mass $(\mathrm{Kg}), \rho$ is its density $\left(\mathrm{Kg} / \mathrm{m}^{3}\right)$, and $r$ is its radius $(m)$.

Of all the solid celestial bodies in the solar system, Earth has the greatest density, radius, and surface gravity. This is good news, because it means that designers will not soon be faced with the seemingly impossible task of insulating against surplus gravity. The problem is instead to supplement or in some way compensate for insufficient gravity, which appears to be far more feasible.

Table 1 lists the major solid bodies, ordered by decreasing surface gravity [1]. (lo, Ganymede, Europa, and Callisto are satellites of Jupiter. Titan is a satellite of Saturn. Ceres is the largest asteroid in the main belt.)

Table 1: Natural Gravity in the Solar System

\begin{tabular}{|lcccc|}
\hline Name & Density & Radius & \multicolumn{2}{c|}{ Surface Gravity } \\
& $\rho$ & $r$ & \multicolumn{2}{c|}{$A_{g}$} \\
& $\left(\mathrm{Kg} / \mathrm{m}^{3}\right)$ & $(\mathrm{m})$ & $\left(\mathrm{m} / \mathrm{s}^{2}\right)$ & $\left(A_{\text {Earth }}\right)$ \\
\hline Earth & 5515 & $6.371 \cdot 10^{6}$ & 9.82 & 1.00 \\
Venus & 5204 & $6.052 \cdot 10^{6}$ & 8.80 & 0.90 \\
Mars & 3933 & $3.390 \cdot 10^{6}$ & 3.73 & 0.38 \\
Mercury & 5427 & $2.440 \cdot 10^{6}$ & 3.70 & 0.38 \\
lo & 3530 & $1.821 \cdot 10^{6}$ & 1.80 & 0.18 \\
Moon & 3344 & $1.738 \cdot 10^{6}$ & 1.62 & 0.17 \\
Ganymede & 1940 & $2.634 \cdot 10^{6}$ & 1.43 & 0.15 \\
Titan & 1881 & $2.575 \cdot 10^{6}$ & 1.35 & 0.14 \\
Europa & 2990 & $1.565 \cdot 10^{6}$ & 1.31 & 0.13 \\
Callisto & 1851 & $2.403 \cdot 10^{6}$ & 1.24 & 0.13 \\
Pluto & 2060 & $1.137 \cdot 10^{6}$ & 0.65 & 0.07 \\
Ceres & 2467 & $0.466 \cdot 10^{6}$ & 0.32 & 0.03 \\
\hline
\end{tabular}

Humanity's total experience in sub-normal surface gravity is merely a few man-days, divided among twelve Apollo Lunar astronauts. Nevertheless, that's enough to confirm that low gravity presents issues in personal mobility: it decreases weight and traction, but not mass or momentum. In a post-flight technical debrief of his Lunar surface experience, Buzz Aldrin commented: "I don't think there is such a thing as running. It's a lope and it's very hard to just walk. You break into this lope very soon as you begin to speed up" [2].

The stiffness and balance of the Apollo extravehicular mobility units (EMUs) further confounded Lunar surface mobility. Nevertheless, the sheer mass of the EMUs - about $81 \mathrm{Kg}$ for the model A7L [3] - may actually have helped by compensating somewhat for the low gravity. In an enclosed shirt-sleeve environment, there may be greater problems with traction and ceiling height. 
Every stride, or "lope", involves vertical as well as horizontal motion. For a vertical leap in a gravitational field, the maximum height and the time to reach that height depend on the energy and mass of the person, and on the gravitational acceleration:

$$
\begin{aligned}
h & =E /\left(m \cdot A_{\mathrm{g}}\right) \\
t & =\sqrt{2 \cdot h / A_{\mathrm{g}}} \\
& =\sqrt{2 \cdot E / m} / A_{\mathrm{g}}
\end{aligned}
$$

where $h$ is the height $(\mathrm{m}), t$ is the time $(\mathrm{s}), E$ is the energy $\left(\mathrm{Kg} \cdot \mathrm{m}^{2} / \mathrm{s}^{2}\right), m$ is the person's mass $(\mathrm{Kg})$ and $A_{\mathrm{g}}$ is the gravitational acceleration $\left(\mathrm{m} / \mathrm{s}^{2}\right)$. In an environment with a surface gravity $n$ times Earth's, assuming equal energy and mass for the person:

$$
\begin{aligned}
A_{\text {planet }} & =n \cdot A_{\text {Earth }} \\
h_{\text {planet }} & =h_{\text {Earth }} / n \\
t_{\text {planet }} & =t_{\text {Earth }} / n
\end{aligned}
$$

Where $n$ is small, $h$ and $t$ are correspondingly large.

It may be argued that, in a low-gravity environment, one should adapt one's gait, use less energy, or redirect one's energy to limit the vertical component. However, maintaining adequate floor traction may require a significant vertical push. Stepping lightly reduces traction and increases the time required to accelerate and decelerate horizontally. Besides taxing one's patience, the resultant slow motion may have an adverse effect on one's performance, especially in emergencies.

In fact, if one applied the same foot force for the same time in a low-gravity environment as one was accustomed to doing on Earth, the energy of the stride would be greater than on Earth. Less of the force would be spent in fighting gravity, and more of it would go into accelerating the body upward.

A precise physical characterization of a human stride depends on many details of physiology, anthropometry, posture, ergonomics, and behavior that are beyond the scope of this paper. Suffice to say that low gravity tends to reduce available floor traction and increase required ceiling heights for bipedal mobility.

MICRO GRAVITY - As alluded earlier, the benefits of natural gravity within an environment vanish if gravity is allowed to accelerate the environment. This is the case in all orbital habitats (not grounded on any significant celestial body), including solar-orbital interplanetary vessels. Three hundred kilometers above the Earth's surface, Earth's gravity continues to act with $91 \%$ of its surface intensity. Orbital inhabitants' sense of weightlessness is due to the lack of resistance to the gravitational acceleration. If gravity actually vanished, they wouldn't orbit at all, but would fly off to infinity. The gravity is apparently "micro" only with respect to the orbital habitat.
Micro gravity presents myriad practical problems. Over-stuffed or poorly packed containers have a jackin-the-box effect when opened. Splashed or spilled water, food crumbs, dropped pencils, and other litter hangs suspended in the air until it settles into a corner or migrates to an air vent. The Skylab shower had to be watertight and drained by a vacuum; users had to plug their noses and breathe through an air hose to avoid inhaling water droplets. (In lieu of a shower, Shuttle astronauts use wet-wipes.) The Shuttle's malfunctioning toilet was a continuing nuisance on early flights. Actions that rely on weight for force, or friction for stability, are nearly impossible to accomplish; workstations must provide alternative means of restraint. Personal mobility requires handholds, making it somewhat clumsy to carry things. To cross an open space greater than arm's reach, a person must shove away from one surface and collide with another. Designers must provide adequate padding and avoid sharp protrusions to avoid injuring the inhabitants [4-5].

These are mostly matters of engineering. With nearly four decades of orbital experience to draw from, space habitat designers and inhabitants have learned to deal adequately with most of them.

In fairness, it must be noted that micro gravity offers some advantages. As a manufacturing environment, it supports mixtures of elements of dissimilar densities without stratification, and the growth of large, pure crystals. Massive equipment moves easily, albeit slowly, requiring effort only to start it and stop it. However, it's questionable whether people should inhabit such an environment, since their very motion disrupts the purity of the micro gravity.

In a micro-gravity habitat, every surface - "wall", "floor", and "ceiling" - is accessible for work, sleep, or storage. Yet, while micro gravitational space may be amorphous and isotropic, the human body is not. Experience has taught that it's beneficial to maintain a consistent vertical reference to avoid disorientation.

The real problems with micro gravity are physiological. Humans evolved to function in a "weighted" environment, in which the upward push of the ground propagates stresses throughout the body. Weightlessness leads to a chain reaction of adverse physiological adaptations. Briefly:

- fluid redistribution [5-6];

- fluid loss [5, 7];

- electrolyte imbalances [6-7];

- cardiovascular changes [4, 7-8];

- $\quad$ red blood cell loss [8-9];

- muscle damage [4, 8-10];

- bone damage [4-9, 11-14];

- hypercalcemia [4-5];

- diminished immune response [5, 7-8];

- thickened bacterial cell membranes [5];

- vertigo and spatial disorientation [4, 15];

- nausea and malaise $[4,8]$;

- loss of exercise capacity [5, 16];

- diminished sense of smell and taste [4-5]; 
- weight loss [5, 16];

- flatulence [5];

- facial distortion [5];

- changes in posture and stature $[5,17]$.

Many of these adaptations don't pose serious problems as long as the inhabitants remain in orbit. Trouble ensues upon the return to a planetary surface. The reentry process itself is especially dangerous, as the apparent gravity may increase rapidly from zero to several times Earth's surface value. After a 237-day mission in 1984, Soviet cosmonauts felt that if they had stayed in space much longer, they might not have survived reentry [7].

Subsequent Soviet and Russian missions have surpassed a year in weightlessness. These longduration missions are milestones of human endurance. But, they are not models for either large-scale space habitation or interplanetary exploration. Crew members must invest increasing amounts of time in grueling, monotonous physical exercise for self preservation, significantly reducing the time available for productive work. In the end, the goal of the mission is little more than survival. After leaving orbit and returning to solid ground, it may take months for the crew to fully readapt to life with gravity.

Although exercise in micro gravity has been somewhat successful at maintaining adequate muscular and cardiovascular fitness, it has been less effective in preventing bone loss. This may be because the treadmills, rowing machines, and bicycle ergometers typically used in orbit don't sufficiently load the skeletal system. Bone minerals are continually deposited where needed and resorbed where not needed, guided by the piezoelectric behavior of bone tissue under stress [9, 11]. To preserve bone structure, Woodard [12], and Keller, Strauss, and Szpalski [13] propose brief periods of high-intensity strength training, rather than long sessions of endurance exercise.

Another attempted countermeasure has been the periodic use of a "penguin suit" specifically designed to stress the skeletal system [6].

There's an ongoing search for medicinal treatments, as alternatives to normal skeletal loading, but these are highly problematic. Dietary mineral supplements may increase the concentration of minerals in the blood, urine, and feces, and exacerbate the risk of developing urinary stones, without adding anything to the bones. Drugs that accentuate bone growth may act inappropriately in micro gravity, due to fluid redistribution as well as the lack of significant pressure points in the skeleton.

ARTIFICIAL GRAVITY - To simulate the experience of natural surface gravity, it's necessary merely to provide an external force equal to what the ground would exert in reaction to gravitational attraction (or some proportion thereof).
For a person at rest on the ground, it's useful to think of the acceleration not simply as zero, but rather as the sum of two equal and opposite accelerations. Natural gravity acts directly on every particle of mass, and tends to accelerate the person downward without propagating stresses within the body (assuming the gravity gradient over the person's height is negligible). The solid ground exerts an equal and opposite acceleration, but it acts directly only on the layer of mass in direct contact with it. Each layer of mass within the body supports all of the layers above, resulting in compression that's greatest at the bottom and diminishes to zero at the top.

Any externally applied acceleration will propagate such stresses within the body. Whether on or off the surface of a planet, the apparent weight of a body is actually due to its non-gravitational acceleration:

$$
\mathbf{W}=-m \cdot\left(\mathbf{A}-\mathbf{A}_{\mathrm{g}}\right)
$$

where $\mathbf{W}$ is the apparent weight vector (directed "down"), $m$ is the mass of the body, A is the total acceleration vector (including gravity and other influences), and $\mathbf{A}_{g}$ is the gravitational acceleration vector. In the special case of standing on the ground, $\mathbf{A}=0$, and the formula reduces to $\mathbf{W}=m \cdot \mathbf{A}_{\mathrm{g}}$. In freefall (or orbit), $\mathbf{A}=\mathbf{A}_{\mathrm{g}}$ and $\mathbf{W}=0$.

Any change in velocity over time, in direction or magnitude, constitutes acceleration. Acceleration is linear or centripetal (or a combination) according to whether it's aligned with the velocity or orthogonal to it.

The effects of constant linear non-gravitational acceleration are virtually indistinguishable from the reaction to natural gravity on a planetary surface. However, integrated over time, linear acceleration leads to ever-increasing speed and displacement. It demands a continuous increase in a body's kinetic energy and precludes it from staying near any particular place. Thus, linear acceleration is not a viable approach to artificial gravity either on a planetary surface or in planetary orbit. It may be applicable to long-duration interplanetary or interstellar flights, if the requisite energy source and propulsion system could be developed. No such system appears on the horizon, however.

In contrast, a body in constant centripetal acceleration rotates at a constant radius and angular rate around a center point. It consumes no energy, because the accelerating force is always orthogonal to the velocity (so the dot product is always zero). The center point of the rotation may be fixed on a planetary surface, or it may be in orbit. In the latter case, gravity accelerates the entire rotating environment, but has no effect on the apparent weights of bodies within the environment (again assuming that the gravity gradient is negligible). Thus, centripetal acceleration is a viable approach to artificial gravity. 
Four parameters characterize rotating environments:

$$
\begin{array}{ll}
\boldsymbol{\Omega} & \text { angular velocity }(1 / \mathrm{s}) \\
\mathbf{r} & \text { radius }(\mathrm{m}) \\
\mathbf{V}_{\mathrm{t}} & \text { tangential velocity }(\mathrm{m} / \mathrm{s}) \\
& =\boldsymbol{\Omega} \times \mathbf{r} \\
\mathbf{A}_{\text {cent }} & \text { centripetal acceleration }\left(\mathrm{m} / \mathrm{s}^{2}\right) \\
& =\boldsymbol{\Omega} \times(\boldsymbol{\Omega} \times \mathbf{r})
\end{array}
$$

With the restriction that $\Omega$ and $\mathbf{r}$ are orthogonal, specifying values for any two of these parameters determines the values of the other two as well [18]. The designer is free to choose any two.

The centripetal acceleration is the nominal "design acceleration" for bodies at rest within the rotating environment. It determines the "up" vector, directed toward the center of rotation. The apparent gravity is equal and opposite to the acceleration.

Unfortunately, centripetal acceleration is an imperfect source of artificial gravity. Because its intensity is proportional to the radius of rotation, there is an effective gravity gradient on the vertical (radial) axis. To keep the gradient small, the radius $r$ must be large.

Furthermore, relative motions within the rotating environment introduce additional accelerations, forces, and torques that one does not encounter in natural gravity. These distort the apparent gravity in proportion to the relative motion.

Relative linear motion in the rotating environment involves Coriolis acceleration in inertial space. This modifies the moving body's apparent weight, adding a component proportional and orthogonal to its relative velocity:

$$
\mathbf{A}_{\mathrm{Cor}}=\mathbf{2} \cdot \mathbf{\Omega} \times \mathbf{v}
$$

where $\mathbf{A}_{\text {Cor }}$ is the Coriolis acceleration, $\boldsymbol{\Omega}$ is the environment's angular velocity in the inertial reference, and $\mathbf{v}$ is the body's relative linear velocity in the rotating reference. In the absence of the required Coriolis force, the body's motion will appear to deviate in the rotating reference as if it was accelerated by some mysterious force. The acceleration is zero for motion parallel to the axis, and maximum for motion in the plane of rotation.

The relative velocity $\mathbf{v}$ may be a parameter of human behavior beyond the designer's control. To keep the Coriolis acceleration small, the environment's angular velocity $\Omega$ must be small.

Also of interest is the ratio of Coriolis to centripetal acceleration. For motion in the plane of rotation:

$$
\begin{aligned}
A_{\text {Cor }} / A_{\text {cent }} & =(2 \cdot \Omega \cdot v) /\left(\Omega^{2} \cdot r\right) \\
& =2 \cdot v / V_{\mathrm{t}}
\end{aligned}
$$

To keep this ratio small, the environment's tangential velocity $\mathbf{V}_{\mathrm{t}}$ must be large.

Relative rotational motion in the rotating environment involves angular acceleration in the inertial reference, and requires an additional torque:

$$
\mathbf{M}=\dot{\mathbf{H}}_{\mathrm{r}}+\boldsymbol{\Omega} \times \mathbf{H}
$$

where $\mathbf{M}$ is the torque, $\boldsymbol{\Omega}$ is the environment's angular velocity in the inertial reference, $\mathbf{H}$ is the body's angular momentum, and $\dot{\mathbf{H}}_{\mathrm{r}}$ is the rate of change of $\mathbf{H}$ with respect to the rotating reference. If the body rotates around a local axis that's not aligned with the environment's rotation, then $\mathbf{H}$ is not aligned with $\boldsymbol{\Omega}$ and a component of torque emerges on an axis perpendicular to both. For example, a person turning sideways around his $z$ axis, in an environment rotating around his $x$ axis, experiences a torque around his $y$ axis. Moreover, the effect of this torque on the vestibular organs produces an illusion of rotation about the $y$ axis [19-20]. His rotation within the environment, and the environment's rotation in inertial space, are said to be "cross-coupled". These crosscoupled rotations wreak havoc on the vestibular system and are a major cause of motion sickness. To keep the cross-coupling small, the environment's angular velocity $\Omega$ must be small.

\begin{tabular}{|c|c|c|c|c|}
\hline Author & $\begin{array}{c}\text { Min. } \\
r \\
(m)\end{array}$ & $\begin{array}{c}\text { Max. } \\
\frac{30 \cdot \Omega}{\pi} \\
(\mathrm{rpm})\end{array}$ & $\begin{array}{c}\text { Min. } \\
V_{\mathrm{t}} \\
(\mathrm{m} / \mathrm{s})\end{array}$ & $\begin{array}{c}\text { Min. } \\
\frac{A_{\text {cent }}}{9.81} \\
\left(A_{\text {Earth }}\right)\end{array}$ \\
\hline Hill \& Schnitzer [21] & 14 & 4 & 6 & 0.03 \\
\hline $\begin{array}{l}\text { Gilruth [22] } \\
\text { "optimum" }\end{array}$ & $\begin{array}{l}12 \\
67\end{array}$ & $\begin{array}{l}6 \\
2\end{array}$ & $\begin{array}{r}6 \\
14\end{array}$ & $\begin{array}{l}0.3 \\
0.3\end{array}$ \\
\hline Gordon \& Gervais [23] & 12 & 6 & 7 & 0.2 \\
\hline Stone [24] & 15 & 6 & 10 & 0.2 \\
\hline Cramer [25] & 22 & 3 & 7 & 0.1 \\
\hline
\end{tabular}

Researchers have studied human adaptation to rotation in Earth-based centrifuges and rotating rooms, and have arrived at various estimates of the "comfort zone" for rotation. Table 2 summarizes some of the estimated limits on four parameters. As is customary, it lists angular velocities in rotations per minute (rpm) and accelerations in multiples of Earth's surface gravity.

Table 2: Comfort Limits for Rotation

Authors often specify limits in terms of other parameters, such as maximum gravity gradient or Coriolis ratio. Some of the values in Table 2 derive from those. Some are the result of satisfying two other constraints simultaneously. In general, the limit values for the various parameters do not occur simultaneously. 
With regard to the maximum angular velocity, the range of values indicates some inconsistency in defining "comfort". The higher values are more appropriately thought of as "tolerance". Graybiel [26] noted that: at $1 \mathrm{rpm}$, even highly-susceptible subjects were symptom-free; at $3 \mathrm{rpm}$, subjects experienced symptoms but weren't significantly handicapped; at $5.4 \mathrm{rpm}$, only subjects with low susceptibility performed well.

With regard to the minimum centripetal acceleration or apparent gravity, the criterion is usually to provide adequate floor traction for mobility. The value from Hill and Schnitzer is an outlier that appears to be an arbitrary bound on their logarithmic graph. The minimum required to preserve health remains unknown.

\section{GRAVITATIONAL SYSTEMS}

The function of a gravitational system, as with other environmental systems, is to control an environmental parameter - gravity - to keep it within acceptable limits for human health and comfort. Here, "gravity" refers to the apparent gravity as perceived through the sensation of weight. Gravity differs from other environmental parameters in at least two respects:

- The naturally occurring value is never too high on any solid celestial body in this solar system, and it's zero in orbit. So, there's never a problem to insulate against or remove surplus gravity. The problem is always to supplement an insufficient natural value.

- In any particular place, or in unpropelled orbit, the natural value is constant.

As with other environmental systems - for example, lighting or heating - gravitational systems may operate on several levels. Various subsystems may address the entire habitat, local areas within the habitat, or even individual inhabitants.

HABITAT GRAVITATIONAL SYSTEMS - The only viable mechanism for producing sustained artificial gravity is centripetal acceleration. With this, the acceleration vector is constant in magnitude, but constantly changing in direction, always pushing toward an axis of rotation. Therefore, the only way to add it to the constant ambient natural gravity and maintain a constant total magnitude is to orient the artificial gravity orthogonal to the natural gravity. The orientation of the axes in Figure 2 has more than symbolic value; it's echoed in the physical configuration of the system shown in Figure 3.

With a constant natural component on the inertial $Z$ axis, and an artificial component proportional to radius rotating in the $X Y$ plane, the slope of the apparent horizontal (normal to the apparent weight) is also proportional to radius. Within the rotating habitat, therefore, the level floor is a paraboloid of revolution.
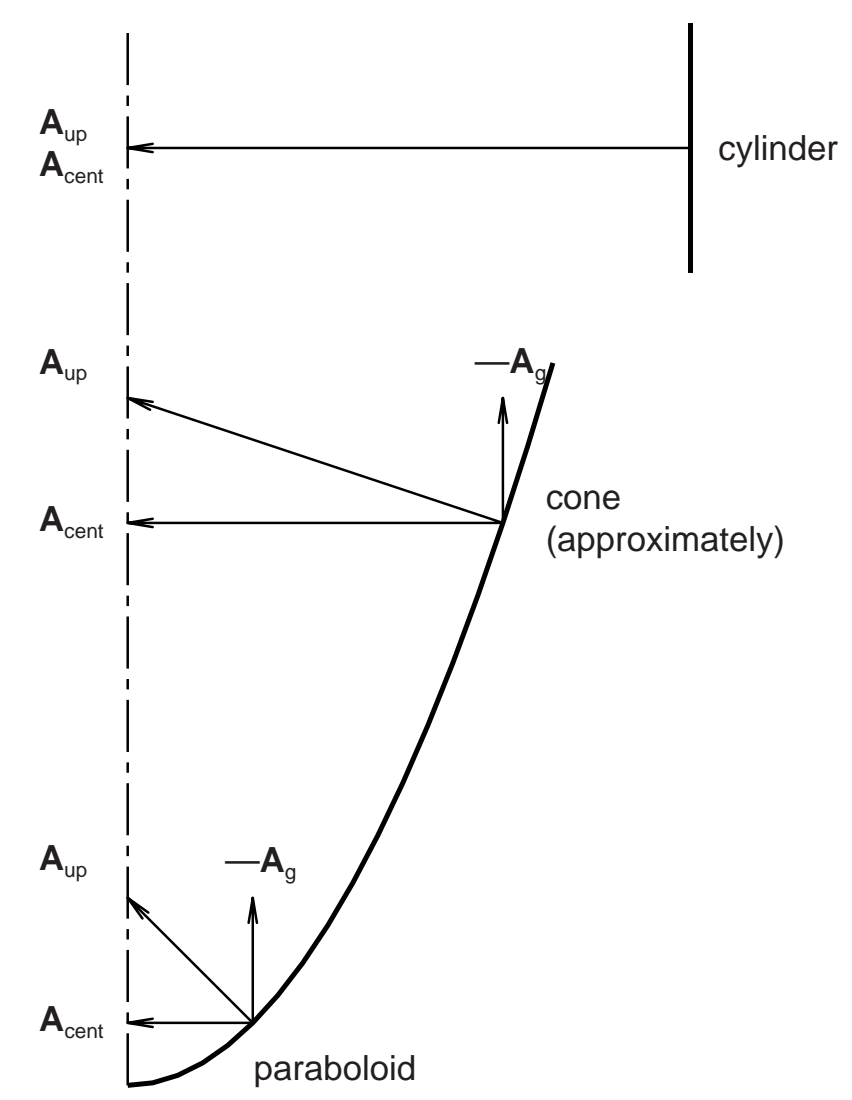

Figure 3: Supplementing Insufficient Natural Gravity with Artificial Gravity

Where the natural component is small with respect to the artificial component, the slope is steep, and a wide floor spans only a small radius between its inside and outside edges. The change of slope across the width of the floor is small, and the paraboloid can be adequately approximated by a slice of a cone.

Where the natural component is zero (in an orbital habitat), the slope is infinite, and the paraboloid degenerates into a cylinder.

To supplement significant but insufficient natural gravity with artificial gravity by rotating the entire habitat, a designer must consider several issues:

Axial Skew - The principal axes of the inhabitant's head are virtually always skew to the habitat's rotation axis. This essentially guarantees that any rotation of the head will cross-couple with the habitat rotation to produce disorienting torques and illusions of rotation about the mutually perpendicular axis.

In contrast, where the natural component is insignificant, the habitat's rotation axis is horizontal in the inhabitant's frame of reference, and aligned with the head's pitch axis when facing prograde or retrograde. This allows at least head pitch without cross-coupling.

Spatial Twist - A corridor around the circumference of the habitat bends on two axes, turning to the side as 


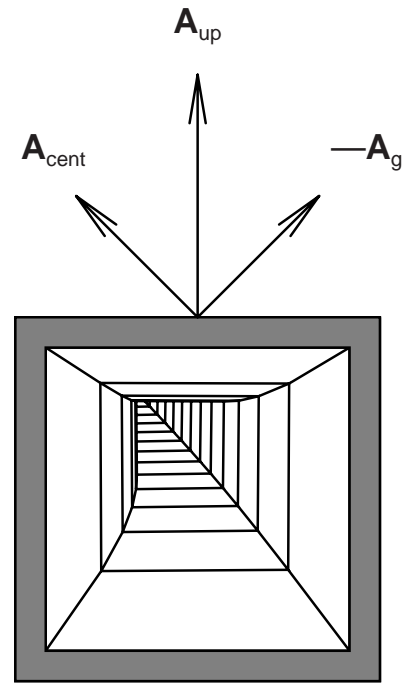

a

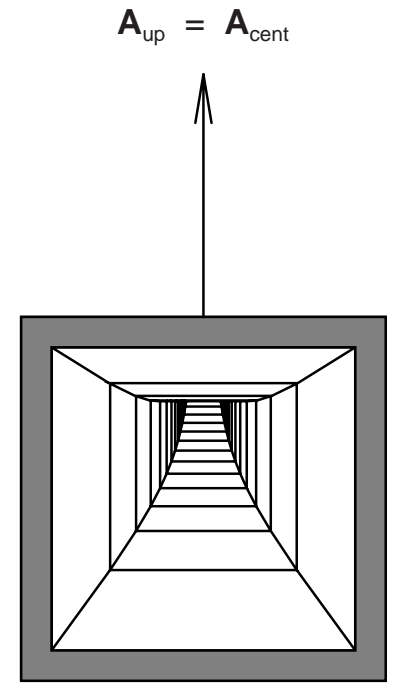

b
Figure 4: Spatial Twist in a Hybrid Natural-Artificial Gravity Environment

it rises, as shown in Figure 4a. Aside from the strange visual impact, this also influences the geometry of components to fit into the volume.

The side twist is less pronounced where the natural gravitational component is small. In a purely artificial gravity environment, it disappears, and the corridor assumes the simpler, somewhat more familiar form of a torus, shown in Figure 4b, bending in pitch but not roll or yaw.

Friction, Work, and Energy - While the centripetal acceleration as such consumes no energy, a large rotating structure resting on a planetary surface will require substantial bearings and continual energy input to overcome friction.

In contrast, a rotating structure in orbit encounters virtually no friction. It requires energy to start and stop the rotation, but very little energy to maintain it. Perturbations from natural gravity gradients and other external influences are small.

FOCUSED GRAVITATIONAL SYSTEMS - Rather than continually rotating the entire habitat, one may rotate certain sections intermittently. Analogous to space heating, task lighting, or simulated sunlight as a therapy for seasonal affect disorder, one may consider something like "task gravity" or "therapeutic gravity". Inasmuch as the principal goal of artificial gravity is to preserve health, the "task" is simply to expose oneself to it.

This may take several forms, but probably involves periodic confinement in a small centrifuge in a dedicated area of the habitat. The required dose of gravity is still unknown, but it's reasonable to expect that shorter exposures may be more intense. To generate significant artificial gravity in a small centrifuge pushes the limits of the comfort zone for rotation. Since it may be difficult to perform useful work or engage in enjoyable recreation during these confined exposures, it may be best to schedule them during sleep.

Experiments with rotating beds indicate that subjects can tolerate much smaller radii and much higher angular velocities than indicated in Table 2 . The inactivity of the subjects and the lack of any performance requirement other than sleep are important factors in extending the comfort zone. Also, it may be serendipitous that the first symptoms of motion sickness often include drowsiness.

Cardús et al. [27-30] have developed and tested an "artificial gravity sleeper", in the form of a flat disk with four cots arranged as radial spokes. Subjects lie with their heads near the center of rotation. The disk rotates up to 30 times per minute to produce Earthintensity artificial gravity at a radius of 1 meter (near each subject's center of mass). In an environment with some significant natural gravity, the cots would seem to tilt, with the head up and the feet down. The smaller the natural gravitational component, the greater the apparent tilt. Where the natural component is zero, subjects would seem to be standing up. Thus, their cardiovascular and skeletal systems may need to work harder during sleep than during wakefulness. In fact, that's precisely the purpose of the rotating bed. It's somewhat ironic that, on Earth, extended bed rest is used to simulate certain aspects of weightlessness, whereas in actual weightlessness, bed rest - on a rotating bed - may be used to simulate weight.

Another approach to "task gravity", proposed by Antonutto, Capelli, and di Prampero [31], is a system whereby inhabitants work for their gravity by riding bicycles around the inside of a cylindrical module.

In all such focused gravitational systems, the main problem is stability. Unless a firm, immovable anchor is available, these systems must be organized into counter-balanced counter-rotating pairs to avoid imparting wobble or rotation to the entire habitat. This is particularly important for orbital habitats. They may require groups of inhabitants to coordinate their sleeping or bicycling activities. This sort of discipline may be acceptable in military, scientific, or commercial "missions", but is less applicable to unstructured social situations such as tourism. Particularly with rotating beds, it would be impractical and disturbing to repeatedly start and stop the rotation of a multi-cot disk to allow individuals to get on or off. Rotating the cots individually would multiply the volume requirement, since each would need to rotate through its own spatial disk. Each cot would also require some counter-balancing mass, though with a bit of planning the individual's personal belongings might suffice.

WEARABLE GRAVITATIONAL SYSTEMS - In engineering the thermal environment, ASHRAE and ISO standards committees have adopted the concept 
of the "clo" - a unit of measure for the thermal resistance of an individual's clothing. Thus, clothing is an integral part of the overall thermal system.

Similarly, it's worth considering whether clothing may be an important part of the overall gravitational system.

Spacesuits tend to be massive, not by design, but as a consequence of the many life-support functions they must perform. One of the problems facing Mars mission planners is to reduce the mass. The Apollo model A7L is considered unsuitable for working in Mars's surface gravity, which is more than twice the Moon's. If possible, one would like to take advantage of the low gravity to accomplish more work in the available time. So, there's little incentive to add any mass to spacesuits beyond what's necessary to provide immediate life support.

A massive suit may compensate somewhat for low natural gravity to provide a healthy load on the musculoskeletal system. But, a suit so massive as to completely compensate for the gravity deficit appears impractical. Such a suit would need to be nearly twice a man's mass on Mars, and five times his mass on the Moon - far more massive than even the model A7L. Containing the bulk and maintaining the balance of such a garment would be formidable tasks. Moreover, inertia is independent of apparent weight. Though it may weigh much less than it would on Earth, it would be just as difficult to accelerate, slowing all of the wearer's motions, regardless of its flexibility. The weight of a massive suit may improve foot traction, but its increased momentum would require proportionally more traction to start and stop, so there appears to be no advantage.

Finally, where natural gravity is the least, and the need for some sort of compensation is the greatest, adding mass is completely ineffective. Zero gravity yields zero weight, regardless of the mass. This approach appears to be a dead-end.

If a garment is to compensate for a lack of gravity, it obviously must not depend on gravity for its effect. One is left with a few techniques, such as the elastic "penguin suit" to stress the skeletal system, and various sorts of "sticky" shoes (magnetic, adhesive, Velcro) to assist traction. These have no effect on the vestibular or cardiovascular systems, and do nothing to mitigate the problems precipitated by the shift of bodily fluids toward the torso and head.

There is little that an individual can do to shield himself from the effects of either too much or too little natural gravity, without reliance on some larger system. Spacesuits accommodate several other vital environmental systems, but their effectiveness as gravitational systems is marginal at best.

\section{CONCLUSION}

While there may be some similarities between gravitational and other environmental systems, there are some profound differences.

Most environmental parameters, such as temperature and humidity, are scalar values. Others, such as illumination and sound, may have some directional aspect, but multiple sources, focused or diffuse, are generally not disorienting. It's straightforward to modify the naturally occurring value by adding, insulating, or extracting.

Gravity is unique in having a strong directional aspect. Evolution has optimized the human body to function in a particular orientation with respect to a single, strong, constant "source". Adding other vectors, or changing their direction, can be quite disorienting.

For rotating habitats, the worst case may be where the natural and artificial components are equal, as this creates the most twisted floor surface and the greatest skew between the inhabitant's principal axes and the habitat's rotation. If the apparent gravity in such an environment is equal in magnitude to Earth's, then the natural and artificial components are each $70 \%$ of Earth's. Such a high natural component may not require any artificial supplement, and it may be better to make up any inadequacy by means other than imposing rotation on the entire habitat. Anyway, among the solid planets in the solar system, only Earth and Venus provide so much natural gravity; most places provide much less.

On a planetary surface with significant natural gravity, the best means of preserving health may be to provide ample recreation space conducive to vigorous, energetic movement. Problems with traction may be avoided by deliberate selection of anti-skid surfaces for floors and footwear.

Where natural gravity is seriously deficient, it may be beneficial to rotate the habitat. Here, the artificial component will be much greater than the natural component, so that the habitat's rotation axis is nearly horizontal in the inhabitant's reference frame. For an inhabitant facing prograde or retrograde, the cross-coupling effects of head pitch will be small.

To provide Earth-intensity gravity on Mars, the artificial-to-natural component ratio would be 2.43:1. On the Moon, the ratio would be 5.97:1. Without experience, it's impossible to predict the comfort limits of such ratios. However, it seems likely that, with a sufficiently large radius and small angular velocity, any ratio could be accommodated.

Rotating the entire habitat may be preferred in orbit, where there is no apparent natural gravity, no solid anchor, and no friction to overcome. In contrast, therapeutic centrifuges or rotating beds may be preferred on celestial bodies where the natural gravity 
is significant, there is a stable anchorage, and friction losses for rotating the entire habitat would be large.

Pharmacology may have a role to play in combating the ill effects of inappropriate gravity, but this is problematic. The very state of gravity may affect the body's biochemical reactions, due to fluid shifts, modified patterns of skeletal stress, and so on.

Natural and artificial gravity are not mutually exclusive. Perhaps, like oil and water, they may not mix well. However, there's very little real experience with such systems, and they should not be ruled out a priori.

\section{REFERENCES}

1. Jet Propulsion Laboratory. "Horizons On-Line Ephemeris System." <telnet://ssd.jpl.nasa.gov:6775>. Table 1 of this paper lists the mean volumetric radius and the "surface gravity" at that radius, assuming spherical geometry.

2. Jones, Eric M., editor. "Apollo Lunar Surface Journal: Apollo 11: Mobility and Photography." <http://www.hq. nasa.gov/office/pao/History/alsj/a11/a11.mobility.html>, revised 4 February 2000.

3. National Air and Space Museum. "NASM - Apollo to the Moon - Apollo Space Suit, Model A7L." <http://www. nasm.edu/galleries/attm/a11.om.ss.1.html>, created July 1999.

4. Connors, Mary M.; Harrison, Albert A.; and Akins, Faren R. Living Aloft: Human Requirements for Extended Spaceflight. NASA Scientific and Technical Information Branch, 1985. Special Publication 483.

5. Oberg, James E.; and Oberg, Alcestis R. Pioneering Space: Living on the Next Frontier. McGraw-Hill, 1986.

6. Gunby, Phil. "Soviet Space Medical Data Grow, Other Nations Joining In." Journal of the American Medical Association, vol. 256, no. 15, p. 2026+, October 17, 1986. American Medical Association.

7. Marwick, Charles. "Physicians Called Upon to Help Chart Future Space Effort." Journal of the American Medical Association, vol. 256, no. 15, p. 2015+, October 17, 1986. American Medical Association.

8. Merz, Beverly. "The Body Pays a Penalty for Defying the Law of Gravity." Journal of the American Medical Association, vol. 256, no. 15, p. 2040+, October 17, 1986. American Medical Association.

9. Woodard, Daniel; and Oberg, Alcestis R. "The Medical Aspects of a Flight to Mars." The Case for Mars, p. 173-180. Edited by Penelope J. Boston. American Astronautical Society, 1984. Paper no. AAS 81-239. Volume 57 of the Science and Technology Series, Advances in the Astronautical Sciences.

10. Wickelgren, Ingrid. "Muscles In Space Forfeit More Than Fibers." Science News, vol. 134, no. 18, p. 277, October 29, 1988. Science Service, Inc.

11. Mohler, Stanley R. "Aging and Space Travel." Aerospace Medicine, vol. 33, p. 594-597, May 1962. Aerospace Medical Association.
12. Woodard, Daniel. "Countermeasures for the Effects of Prolonged Weightlessness." The Case for Mars II, p. 655-663. Edited by Christopher P. McKay. American Astronautical Society, 1985. Paper no. AAS 84-187. Volume 62 of the Science and Technology Series, Advances in the Astronautical Sciences.

13. Keller, T. S.; Strauss, A. M.; and Szpalski, M. "Prevention of Bone Loss and Muscle Atrophy During Manned Space Flight." Microgravity Quarterly, vol. 2, no. 2, p. 89-102, 1992. Pergamon Press.

14. Wickelgren, Ingrid. "Bone Loss and the Three Bears." Science News, vol. 134, no. 26, p. 424-425, December 24, 1988. Science Service, Inc.

15. Covault, Craig. "Spacelab Stresses Life Sciences Study." Aviation Week and Space Technology, vol. 119, no. 13, p. 73-82, September 26, 1983. McGraw-Hill.

16. Raymond, Chris Anne. "Physicians Trade White Coats for Space Suits." Journal of the American Medical Association, vol. 256, no. 15, p. 2033+, October 17, 1986. American Medical Association.

17. Griffin, Brand Norman. "Design Guide: The Influence of Zero-G and Acceleration on the Human Factors of Spacecraft Design." NASA Johnson Space Center, August 1978.

18. Hall, Theodore W. "SpinCalc: an artificial-gravity calculator in JavaScript." <http://www.arch.cuhk.edu.hk/ hall/ag/sw/SpinCalc/>, revised 22 March 2000.

19. Clark, Carl C.; and Hardy, James D. "Gravity Problems in Manned Space Stations." Proceedings of the Manned Space Stations Symposium, April 20-22, 1960, p. 104-113. Institute of the Aeronautical Sciences, 1960.

20. Lally, Eugene F. "To Spin or Not To Spin." Astronautics, vol. 7, no. 9, p. 56-58, September 1962. American Rocket Society.

21. Hill, Paul R.; and Schnitzer, Emanuel. "Rotating Manned Space Stations." Astronautics, vol. 7, no. 9, p. 14-18, September 1962. American Rocket Society.

22. Gilruth, Robert R. "Manned Space Stations - Gateway to our Future in Space." Manned Laboratories in Space, p. 1-10. Edited by S. Fred Singer. Springer-Verlag, 1969.

23. Gordon, Theodore J.; and Gervais, Robert L. "Critical Engineering Problems of Space Stations." Manned Laboratories in Space, p. 11-32. Edited by S. Fred Singer. Springer-Verlag, 1969.

24. Stone, Ralph W. "An Overview of Artificial Gravity." Fifth Symposium on the Role of the Vestibular Organs in Space Exploration, p. 23-33. NASA Scientific and Technical Information Division, 1973. Special Publication 115: proceedings of a symposium held in 1970.

25. Cramer, D. Bryant. "Physiological Considerations of Artificial Gravity." Applications of Tethers in Space, vol. 1, p. 3.95-3.107. Edited by Alfred C. Cron. NASA Scientific and Technical Information Branch, 1985. Conference Publication 2364: proceedings of a workshop held in Williamsburg, Virginia, June 15-17, 1983. 
26. Graybiel, Ashton. "Some Physiological Effects of Alternation Between Zero Gravity and One Gravity." Space Manufacturing Facilities (Space Colonies): Proceedings of the Princeton / AIAA / NASA Conference, May 7-9, 1975, p. 137-149. Edited by Jerry Grey. American Institute of Aeronautics and Astronautics, 1977.

27. Cardús, David; Diamandis, Peter; McTaggart, Wesley G.; and Campbell, Scott. "Development of an Artificial Gravity Sleeper (AGS)." The Physiologist, vol. 33, no. 1, supplement, p. S112-S113, 1990. American Physiological Society.

28. Cardús, David; McTaggart, Wesley G.; and Campbell, Scott. "Progress in the Development of an Artificial Gravity Simulator (AGS)." The Physiologist, vol. 34, no. 1, supplement, p. S224-S225, 1991. American Physiological Society.

29. Cardús, David; and McTaggart, Wesley G. "The Cardiovascular Response to the AGS." The Physiologist, vol. 36, no. 1, supplement, p. S155-S157, 1993. American Physiological Society.

30. Houtchens, C. J. "Artificial Gravity." Final Frontier, vol. 2, no. 3, p. 28+, June 1989. Final Frontier Publishing Company.
31. Antonutto, G.; Capelli, C.; and di Prampero, P. E "Pedalling in Space as a Countermeasure to Microgravity Deconditioning." Microgravity Quarterly, vol. 1, no. 2, p. 93-101, 1991. Pergamon Press.

\section{CONTACT}

Theodore W. Hall completed his doctoral degree in architecture at the University of Michigan in 1994. He is currently a research officer in the Department of Architecture at the Chinese University of Hong Kong.

Postal address: Theodore W. Hall; Department of Architecture; Chinese University of Hong Kong; Sha Tin, Hong Kong, China.

e-mail: twhall@cuhk.edu.hk

\section{ADDITIONAL SOURCES}

http://www.arch.cuhk.edu.hk/ hall/ag/ 